\title{
Bilateral axillary skin fold flaps used for dorsal thoracic skin wound closure in a dog
}

\author{
B G Nevilla
}

\begin{abstract}
A 10-year-old greyhound-cross dog was presented with a large, chronic skin wound extending over the interscapular region. The substantial skin defect was closed by making use of bilateral axillary skin fold flaps. It was possible to elevate the 2 skin flaps sufficiently to allow them to meet at the dorsal midline and thus facilitate complete closure of a large and awkwardly positioned wound. Small dorsal areas of the skin flaps underwent necrosis, but the resulting defects were closed without difficulty in a subsequent procedure. To the author's knowledge, this is the 1st clinical report of the use of bilateral axillary skin fold flaps in this fashion and describes an additional use of a versatile skin flap procedure.

Keywords: axillary skin fold flap, bilateral, dog, dorsal thoracic wound.

Nevill B G Bilateral axillary skin fold flaps used for dorsal thoracic skin wound closure in a dog. Journal of the South African Veterinary Association (2010) 81(1): 58-61 (En.). Department of Companion Animal Clinical Studies, Faculty of Veterinary Science, University of Pretoria, Pivate Bag X04, Onderstepoort, 0110, South Africa.
\end{abstract}

\section{INTRODUCTION}

Skin wounds are commonly seen in veterinary practice. For the most part these can be repaired relatively simply with a combination of undermining, apposition and suturing. These procedures may be primary or delayed as indicated to allow for débridement and granulation ${ }^{15}$. Large skin wounds can be more challenging to repair if it is not possible to close the wound with the available surrounding skin. A wide variety of reconstructive options are available to surgeons faced with difficult skin wounds. These include skin stretching or recruitment, free skin grafts, microvascular free tissue transfer and a variety of skin flaps ${ }^{6,15,16}$. Dogs and cats tend to have mobile skin that lends itself to pedicle or flap grafting procedures ${ }^{12,14}$. The axillary skin fold flap has been well described, particularly by Hunt ${ }^{9,10}$. This report describes the simultaneous bilateral elevation of axillary skin fold flaps to close a large, dorsal thoracic skin wound.

\section{CASE HISTORY}

A 10-year-old female greyhound cross was referred to the Onderstepoort Veterinary Academic Hospital for repair of a large, ovoid dorsal thoracic skin defect. The cause of the wound was unknown and had occurred about 3 weeks previously. At the dorsal interscapular region,

${ }^{a}$ Department of Companion Animal Clinical Studies, Faculty of Veterinary Science, University of Pretoria, Pivate Bag X04, Onderstepoort, 0110 South Africa. E-mail: bruce@ ovation.co.za

Received: June 2009. Accepted: January 2010. the wound was about $20 \mathrm{~cm}$ in extent craniocaudally and then tapered to approximately the level of the shoulder joints on both sides (Fig. 1). Wound débridement and suturing had been undertaken twice by the referring practice but the wound had dehisced after each attempt. On presentation, the dog was found to be in good physical condition despite a mild regenerative anaemia, neutropaenia and hypoalbuminaemia. There was substantial contraction of the wound margins and minimal granulation tissue present on the wound bed. Initial conservative treatment entailed daily flushing with ringer's lactate (Ringer Lactate, Intramed, South Africa) and protective bandaging. Cephalexin (Cephalexin Capsules, Alliance Pharma, South Africa) was administered orally at $20 \mathrm{mg} / \mathrm{kg}$ twice daily and continued for 1 week. An attempt was made to recruit additional skin with the use of skin stretchers made from adhesive pads and elastic strips ${ }^{8}$. This was not rewarding as the skin surrounding the wound appeared to have little remaining elasticity. It was decided to employ bilateral axillary skin fold flaps to enable wound closure.

After anaesthetic induction, the hair was clipped widely around both donor and recipient sites, which were then prepared for aseptic surgery. Skin flaps were elevated from both axillary folds in turn. The dog was placed in left lateral recumbency for elevation of the flap from the right fold and then turned to right lateral recumbency to elevate the left fold. The skin of the axillary fold was first pulled caudally to ascertain the maximum amount of skin that could be harvested and still allow closure without excessive tension. A sterile skin marker was used to outline the anticipated skin incisions. The flap thus outlined was elevated and the lateral flap incision extended further dorsally to meet the cranial margin of the

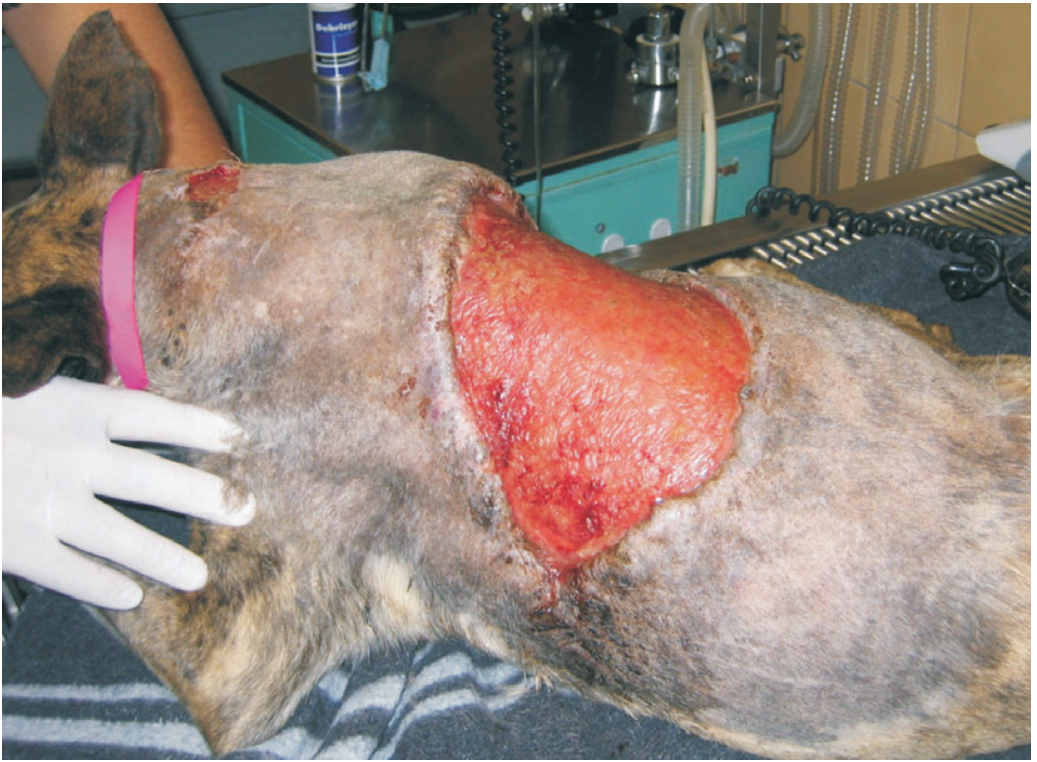

Fig. 1: Appearance of the wound on presentation. 
skin defect. The ventral attachment of the axillary fold to the body wall was retained, forming the base of the flap (Fig. 2). The elevated flap was unfolded and advanced caudodorsally into the skin defect of the corresponding side (Fig. 3a-c). The recipient wound bed was prepared by trimming the skin edges and lavaging with ringer's lactate. The flap was then sutured into position using 4/0 monofilament nylon (Ethilon, Ethicon, South Africa) in a simple interrupted pattern. The procedure was repeated on the opposite side after repositioning the patient. The 2 skin flaps met and were sutured together at the dorsal midline. Modified closed suction drains made out of small feeding tubes and $20 \mathrm{~m} \ell$ syringes were inserted subcutaneously in both recipient sites and maintained for 3 days $^{3}$. The wounds were covered by light protective bandaging for 2 days. Postoperative analgesia was supplied by a $3 \mu \mathrm{g} / \mathrm{kg} / \mathrm{hr}$ transdermal fentanyl patch (Durogesic, Janssen Pharmaceutica, South Africa) and carprofen (Rimadyl, Pfizer, South Africa) given at $2.2 \mathrm{mg} / \mathrm{kg}$ twice daily for 5 days. The donor sites healed uneventfully. Both flaps exhibited some postoperative congestion and oedema especially dorsally. Areas of necrosis approximately $20 \mathrm{~mm} \times 20 \mathrm{~mm}$ developed in both flaps - caudodorsally on the left side and craniodorsally on the right. These were excised and the resulting defects closed without difficulty in a subsequent procedure undertaken a week after the 1st one. Thereafter healing progressed well with sutures removed after 10 days (Fig. 4).

\section{DISCUSSION}

Large skin wounds can be challenging to repair and more than one method of repair may potentially be successful ${ }^{8}$. Skin stretching was initially attempted as a means of closing or at least reducing the size of the wound in this patient. The lack of skin elasticity encountered was unexpected and may have been the consequence of the previous attempts to close the wound. A free skin graft was considered as a potential method of closure. However, the strenuous requirements of postoperative care, including the need for secure postoperative immobilisation, the risk of graft necrosis secondary to infection, and the size of the potential donor site, made this option less appealing $^{13,15}$. Microvascular tissue transfer techniques could potentially have been employed, but require particularly specialised equipment and skills ${ }^{6}$. The proximity of the axillary folds to the wound and the generous size of the folds in this particular dog, motivated the decision to use the axillary skin fold flaps. Skin flaps or pedicle

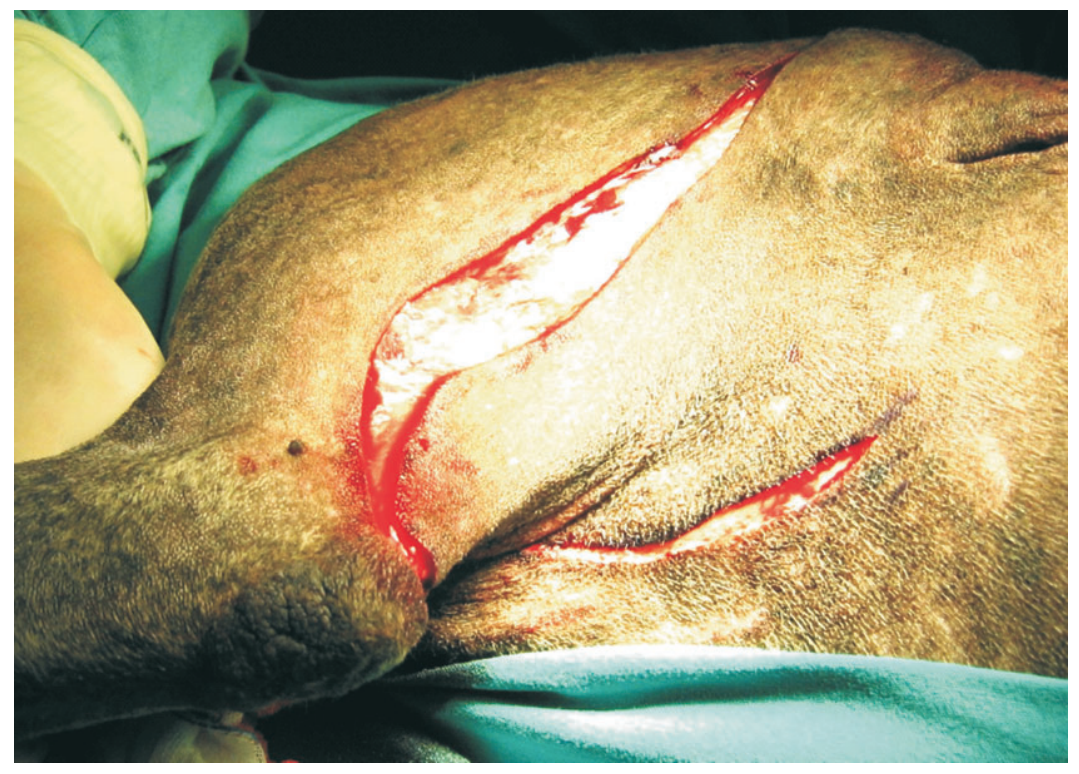

Fig. 2: Lateral and medial incisions delineating the skin fold flap. The 2 incisions met just proximal to the elbow. The lateral incision was extended dorsally to meet the cranial margin of the wound.

grafts are a useful means of transferring skin from one region of the body to another where there is a skin deficit. Possibly the best-known skin flap in

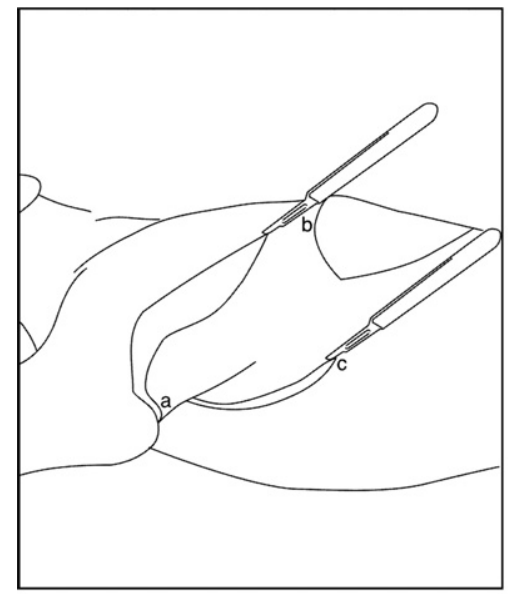

a)

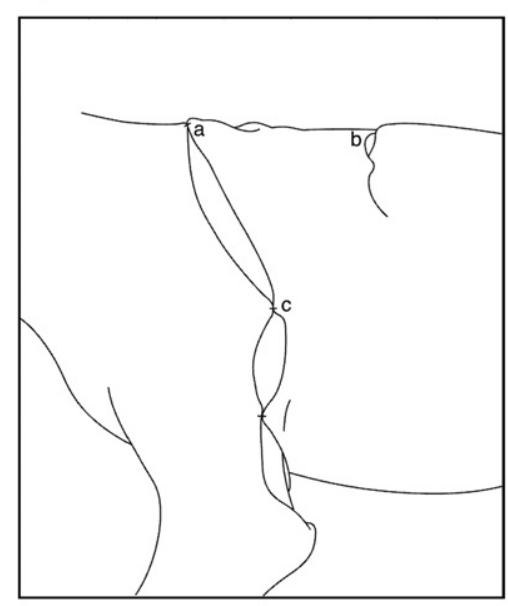

c) domestic pets is the superficial caudal epigastric flap and bilateral elevation of this flap has been described ${ }^{13}$. The pedicle, or base of a skin flap, provides ongoing

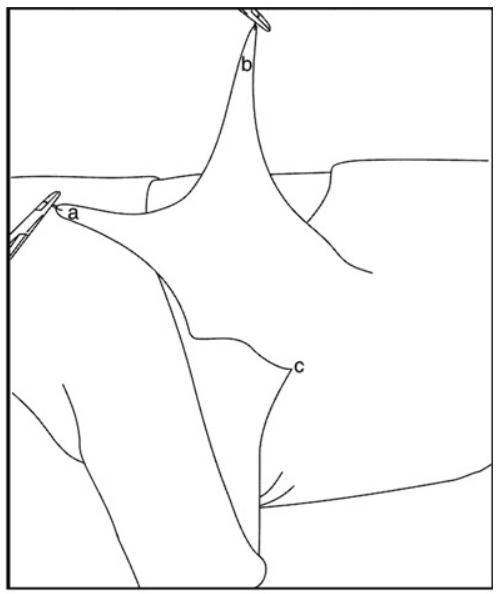

b)
Fig. 3: Schematic illustration of sequential flap outline (a), elevation and transposition (b) and final positioning (c). The procedure was repeated bilaterally with the 2 flaps meeting on the dorsal midline. 


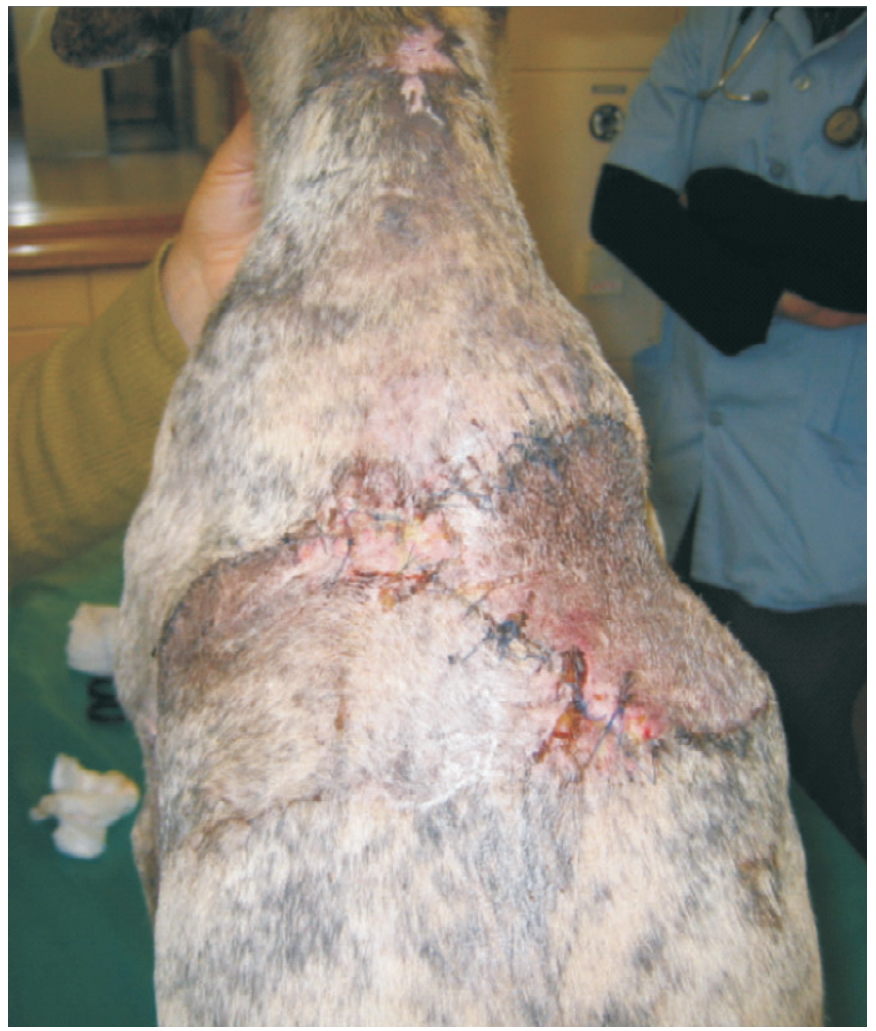

Fig. 4: Wound appearance two weeks after the second procedure. The skin flaps met and were sutured together dorsally.

blood supply to the flap despite the latter having been elevated from its original site ${ }^{15,17}$. The axillary folds provide an easily accessible source of skin that can potentially be used to close wounds in a variety of positions in the region. An axillary fold can be regarded as having a medial and lateral attachment to the front leg and a dorsal and ventral attachment to the body wall (Fig. 5a,b). Any 3 of these attach- ments can be mobilised while the remaining attachment is left intact to form the base of the flap ${ }^{8,9,11,12}$. Maintaining a body wall attachment may lead to better flap survival ${ }^{5}$. Depending on where the base is positioned, it is possible to cover areas ranging from mid-radius to the lateral thoracic wall ${ }^{5,11,12,17}$. The amount of skin available to harvest depends on the particular conformation of the patient, but a surprisingly large flap results when the medial and lateral portions of the fold are flattened out. Limb function and range of motion are not adversely affected ${ }^{4}$. Cats tend to have very well developed skin folds ${ }^{7,11}$.

The mobilised axillary skin fold flap is generally regarded as a subdermal plexus flap and as such, not as robust as an axial pattern flap ${ }^{11,15}$. Accordingly, it is advised that the base of the flap (i.e. the width) approximates $50 \%$ of the length of the flap to ensure adequate perfusion from the subdermal plexus ${ }^{5,10}$. Recent work has, however, suggested that the axillary skin fold flap may be an axial pattern flap supplied by the lateral thoracic artery if the flap is carefully elevated. Very good survival rates, equivalent to those reported for axial pattern flaps, have been obtained $^{2,12}$. Careful mobilisation at the base of the flap is thus required to minimise disruption to flap blood supply which could in turn lead to variable degrees of flap necrosis. Other important potential reasons for flap necrosis include kinking, haematoma formation and infection. Careful preoperative planning and atraumatic surgical technique are required to minimise these problems ${ }^{12,15}$.

Although it was not difficult to reach the dorsal midline with the elevated flaps, some difficulty was experienced in spreading tension equally between the 2 flaps because they were created and sutured into position sequentially and the patient was in lateral recumbency. It may have been preferable to create both flaps first and then move them into position dorsally at the same time with the patient posi-

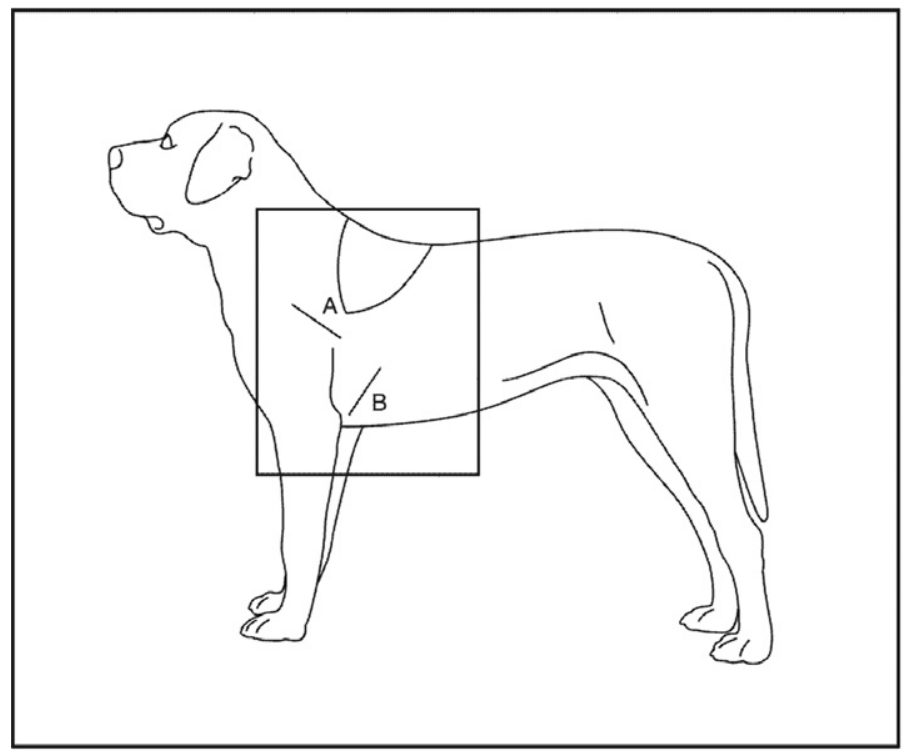

a)

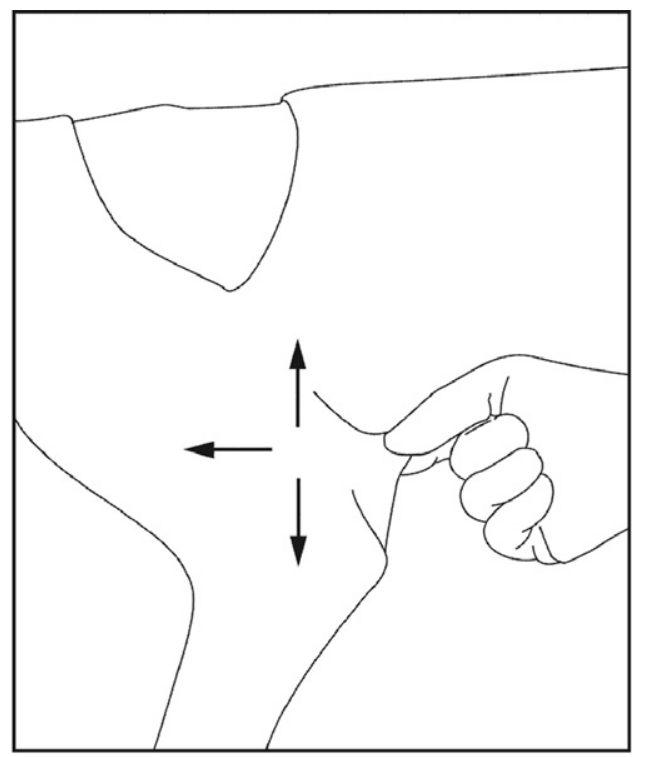

b)

Fig. 5: a, The axillary fold is situated at the caudal aspect of the front limb immediately proximal to the elbow. It has medial and lateral attachments to the front limb and dorsal $(A)$ and ventral $(B)$ attachments to the body wall. b, If the axillary fold is pulled caudally, it is possible to ascertain how much skin can be harvested and still allow tension-free closure. Depending which attachment is retained, it is possible to move the flap in a variety of directions, covering areas from mid-radius, sternum and lateral body wall. 
tioned sternally. The practical implications of repeatedly repositioning the patient and still maintaining appropriate surgical asepsis may make this difficult. Postoperative care in all skin flap procedures should include adequate analgesia as these procedures are very painful. Postoperative cage confinement will also help limit undesirable movement and tension on the skin flaps. Drains are an important consideration as they help to limit dead space formation and fluid accumulation. Closed drains are generally preferable to open drains as they are associated with lower wound infection rates and are easy to make, insert and keep clean ${ }^{1}$. Soft protective bandaging is appropriate for a few days postoperatively, although bandaging should be used with caution as inappropriate pressure on skin flaps may compromise an already tenuous blood supply ${ }^{9}$

Skin flaps are a useful means of transferring skin on the body but do require careful preoperative planning, meticulous surgical technique and appropriate postoperative care. The axillary skin fold flaps are particularly versatile within their range of rotation and bilaterally elevated axillary skin fold flaps can potentially be used to close large dorsal thoracic skin defects.

\section{ACKNOWLEDGEMENTS}

The author would like to acknowledge

Dr Peter Turner's assistance in managing this case.

\section{REFERENCES}

1. Baines S J 1999 Surgical drains. In Fowler D, Williams J M (eds) BSAVA manual of canine and feline wound management and reconstruction. British Small Animal Veterinary Association, Cheltenham: 54-57

2. Brinkley C H 2007 Successful closure of feline axillary wounds by reconstruction of the elbow skin fold. Journal of Small Animal Practice 48: 111-115

3. Dernell W S 2006 Initial wound management. Veterinary Clinics of North America, Small Animal Practice 713-738

4. Fowler D 1999 Tension relieving techniques and local skin flaps. In Fowler D, Williams $\mathrm{J}$ M (eds) BSAVA manual of canine and feline wound management and reconstruction. British Small Animal Veterinary Association, Cheltenham: 59-68

5. Fowler D 2006 Practical patches for worrisome wounds: subdermal plexus flaps. Proceedings of the North American Veterinary Conference, Orlando USA, 7-11 January 2006 1461-1462

6. Fowler J D, Miller C W, Bowen V, Johnston G H 1987 Transfer of free vascular cutaneous flaps by microvascular anastomosis. Results in six dogs. Veterinary Surgery 16: 446-450

7. Gray M J 2005 Chronic axillary wound repair in a cat with omentalisation and omocervical skin flap. Journal of Small Animal Practice 46: 499-503

8. Hedlund C S 2006 Large trunk wounds. Vet- erinary Clinics of North America, Small Animal Practice 36: 846-872

9. Hunt G B 2002 Use of skin folds for reconstructive surgery in the dog. Proceedings of the 27th WSAVA Congress, Granada, Spain, 3-6 October 2002

10. Hunt G B 2006 Skin fold advancement flaps for trunk reconstruction. Proceedings of the North American Veterinary Conference, Orlando USA, 7-11 January 2006: 1405-1407

11. Hunt G B 1995 Skin fold advancement flaps for closing large sternal and inguinal wounds in cats and dogs. Veterinary Surgery 24: 172-175

12. Hunt G B, Tisdall P L C, Liptak J M, Beck J A, Swinney G R, Malik R 2001 Skin-fold advancement flaps for closing large proximal limb and trunk defects in dogs and cats. Veterinary Surgery 30: 440-448

13. Mayhew P D, Holt D E 2003 Simultaneous use of bilateral caudal superficial epigastric axial pattern flaps for wound closure in a dog. Journal of Small Animal Practice 44: 534-538

14. Pavletic M 1990 Skin flaps in reconstructive surgery. Veterinary Clinics of North America, Small Animal Practice: 80-103

15. Pavletic M 2003 Atlas of small animal reconstructive surgery (2 edn). W B Saunders, Philadelphia

16. Swaim S F, Henderson R A 1990 Small animal wound management. Lea and Febiger, Philadelphia

17. Vasconcellos C H, Matera J M, Dagli M L Z 2005 Clinical evaluation of random skin flaps based on the subdermal plexus secured with sutures or sutures and cyanoacrylate adhesive for reconstructive surgery in dogs. Veterinary Surgery 34: 59-63 